\title{
Gorlin Syndrome Presentation and the Importance of Differential Diagnosis of Skin Cancer: A Case Report
}

\author{
Ravina Sanghera ${ }^{1}$ BSc. Pharm, PharmD, Parbeer Singh Grewal ${ }^{2}$ MD, FRCPC \\ ${ }^{1}$ Faculty of Pharmacy and Pharmaceutical Sciences, University of Alberta, Edmonton, Canada and ${ }^{2}$ Faculty of Medicine, \\ Department of Dermatology, University of Alberta, Edmonton, Alberta.
}

Received, August 27, 2018; Accepted, September 4, 2018; Published, September 7, 2018.

\begin{abstract}
In a busy community practice, clinical skin findings can often be misinterpreted. Skin cancers can sometimes mimic rashes like psoriasis, eczema or prurigo nodularis in both appearance and symptoms. Gorlin syndrome is one such genetic syndrome, characterized by the eruption of multiple and early onset basal cell carcinomas (BCCs), which can be mistaken for a rash. We describe a 68-year-old female who presented to the dermatology office with a previous history of over 30 BCCs that had been previously biopsied and/or surgically removed. However, the patient had been lost to follow up for several years and had not been seen by a skin specialist. In the interim, she had been misdiagnosed as having eczematous or psoriatic lesions by primary care providers. Patients with Gorlin syndrome are even harder to diagnose as their skin cancers often do not possess the classic features associated with a basal cell or squamous cell carcinoma. When in doubt, and especially if failing topical therapy, patients presenting with dermatological lesions should be properly referred to a specialist for further assessment and workup.
\end{abstract}

\section{INTRODUCTION}

Gorlin syndrome is a genetic syndrome characterized by the eruption of multiple and early onset basal cell carcinomas (BCCs). Synonyms for Gorlin syndrome include Basal Cell Nevus Syndrome (BCNS), Gorlin-Goltz syndrome and nevoid basal cell carcinoma (1). These lesions begin to develop during adolescence or early adulthood and are characterized by numerous BCCs, along with skeletal, ophthalmologic and neurologic abnormalities (2).

Prevalence of Gorlin syndrome is estimated at 1 per 40,000-60,000. The disease affects men and women in rather equal distribution (1:1.3). Although the disease affects all races, African American and Asians represent only $5 \%$ of cases and more often are incidentally diagnosed with extracutaneous findings, such as odontogenic keratocysts, as compared to the development of BCCs alone (2). While more than 1 million new cases of BCCs are diagnosed each year in the United States, less than 1 percent of these skin cancers are related to Gorlin syndrome (3).

Mutations in patched-1 (PTCH1), a tumor suppressor gene located on chromosome 9q22.3q31, prevent the production of the patched-1 receptor or lead to the production of an abnormal version of the receptor. The altered or missing patched-1 receptor cannot effectively suppress cell growth and division. The loss of additional genes in the deleted region of chromosome 9 may result in features that are not typically associated with Gorlin syndrome which include delayed development, intellectual disability and overgrowth of the body (macrosomia).

Gorlin syndrome is inherited in an autosomal dominant pattern where, in most cases, an affected individual inherits the mutation from an affected parent. Other cases result from new mutations in the PTCH1 gene and occur in individuals with no family history of the disorder.

\section{OBSERVATION}

A 68-year-old female presented to the dermatology office for an assessment. The patient had presented with a preceding history of over $30 \mathrm{BCCs}$ in the past that had been previously biopsied and/or surgically removed. However, the patient had been lost to follow up for several years and had not seen a skin specialist. Her presenting complaint was of an itchy

Corresponding Author: Ravina Sangera-Grewal, Faculty of Pharmacy and Pharmaceutical Sciences, University of Alberta, Edmonton, Canada, E-mail: ravina@ualberta.ca 
and irritating rash on her forearms and torso that she admits to having scratched quite extensively. Her dermatological presentation was misdiagnosed as eczematous or psoriatic lesions by various community practitioners and treated accordingly with various topical preparations. On examination it was noticed that she had about 30-40 erythematous, scaly, excoriated papules and plaques scattered over her arms, torso and face, the largest measuring up to $2 \mathrm{~cm}$ in size. Upon closer examination with a dermatoscope, many of the lesions were noticed to have rolled borders and unusually ulcerated bases consistent with BCCs. The patient's presentation was now correctly re-interpreted and diagnosed as Gorlin's syndrome. Management options were discussed, including surgery, radiation, chemotherapy and local therapy. Upon further discussion the patient declined surgery or chemotherapy and instead chose to undergo treatment with aggressive liquid nitrogen cryotherapy and topical 5-flurouracil cream.

\section{DISCUSSION}

Non-melanoma skin cancers (NMSCs) classically appear as a solitary, erythematous, non-healing, ulcerated or bleeding papules, plaques or nodules, with a pearly border or arborizing telangiectasia. The lesions have often persisted and possibly enlarged slowly over weeks or months. However, to a patient or practitioner, they can non-classically appear as a 'rash' such as eczema, psoriasis or prurigo nodularis. Often this is the case because the presenting lesion can be itchy or irritated, leading a patient to scratch the area repetitively and cause self-inflicted excoriations or small ulcerations within the nodule or plaque. Patients are often mistakenly treated with topical steroids or vitamin D analogues or antifungals for weeks or months until a correct diagnosis is made. The cancer can essentially be masked due to extensive excoriations and inflammation around the area which mimics the presentation of the dermatological conditions as mentioned.

All health care practitioners assessing the skin, including pharmacists, physicians and nurses, need to be aware that skin cancers can be both pruritic and non-pruritic. If a patient presents to the health care practitioner with a solitary, eczematous- or psoriaticappearing lesion, that is unresponsive to local topical therapy, they should be promptly referred for further assessment and a biopsy. Patients with Gorlin syndrome are even harder to diagnose as their skin cancers often do not possess the classic features one associates with a basal cell or squamous cell carcinoma - such as a pearly surface or arborizing or corkscrew telangiectasia. These afflicted patients will have multiple open sores or excoriated lesions mimicking a 'rash' as well. Practitioners need to have a very high index of suspicion for skin cancers in patients with any strong personal history of skin cancer, history of sun exposure and sun burns, and genetic disorders that predispose them to skin cancers such as oculocutaneous albinism, xeroderma pigmentosum, epidermolysis bullosa, lynch syndrome, neurofibromatosis, cowden syndrome and familial atypical mole melanoma syndrome.

Patients with a strong index of suspicion for skin cancer should be questioned regarding previous history of skin cancers, family history of skin cancers and associated features. For example, patients with Gorlin syndrome can often develop palmar pits and skeletal problems. In these instances, patients should be referred to a dermatologist for biopsy and further management. Patients with a family history or suspected history of genetic cancer syndromes should also be referred to a general practitioner for further screening and a geneticist for additional testing.

\section{CONCLUSION}

Health care practitioners always need to have an awareness and concern for potentially serious underlying illness such as skin cancer. Skin cancers can sometimes mimic rashes like psoriasis, eczema or prurigo nodularis, in both appearance and symptoms. When uncertain of a definitive diagnosis, or if patients are unresponsive to topical therapy, practitioners should refer patients for further assessment and workup through their general practitioner or dermatologist.

\section{REFERENCES}

1. Basal cell naevus syndrome. In: DermNetNZ. 2018 [cited August 2018]. Available from: https://www.dermnetnz.org/topics/basal-cell-naevussyndrome/

2. Spiker AM, Ramsey ML. Gorlin Syndrome (Basal Cell Nevus) [Updated 2017 Feb 8]. In: StatPearls [Internet]. Treasure Island (FL): StatPearls Publishing; 2018 Jan-. Available from: https://wwwncbi-nlm-nih- 
gov.login.ezproxy.library.ualberta.ca/books/NBK43 0921/

3. Gorlin syndrome [Internet]. In: U.S. National Library of Medicine. 2012 [cited August 2018]. Available from:https://ghr.nlm.nih.gov/condition/gorlinsyndrome\#statistics

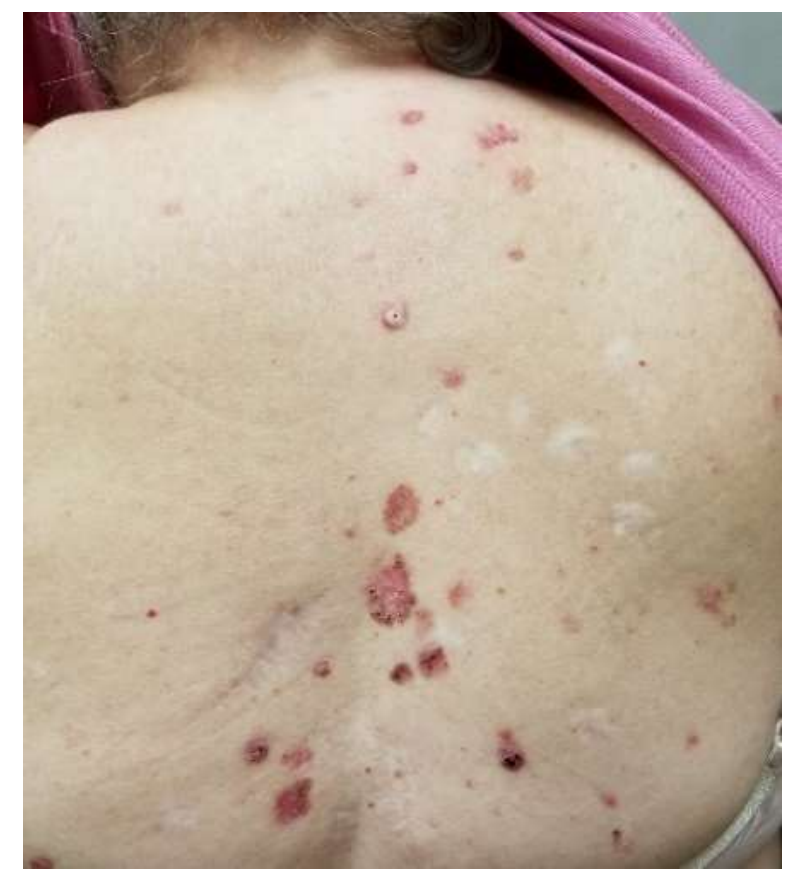

Image 1.168 y.o. female with Gorlin Syndrome. 